\section{PERNICIOUS ANAEMIA.}

Preliminary Note on the After-Results op

Treatment with Liver.

BY

JOHN F. WILKINSON, M.B., M.Sc., Ph.D., F.I.C., DIRECTOR OF THE LABORATORY FOR CLINICAL INVESTIGATIONS AND RESEARCH, MANCHESTER ROYAL INFIRMARY ;

AND

WILliam BROCKBaNK, M.A., M.D.Cantab., M.R.C.P., HONORARY PHYSICIAN, THE MANCHESTER VICTORIA MEMORIAL JEWISH HOSPITAL.

(From the Laboratory for Clinical Investigations and Research, Manchester Royal Infirmary.)

We have been investigating the after-results of all cases of pernicious anaemia admitted to the Manchester Royal Infirmary during and since 1925, and are publishing our preliminary observations in the hope that they will be of interest to other workers investigating this disease.

The cases fall naturally into two sections: (a). Those that received the usual treatment of arsenic and hydrochloric acid before the introduction of the Murphy-Minot diet. (b) Those that received the liver treatment, often in addition to the drugs mentioned. The total number of cases studied has been 80 , of which 47 belong to the first group and 33 to the second. Briefly our observations are as follows.

1. Of the 33 patients who left the hospital on the liver diet about 50 per cent. are very well and 15 per cent. in good health except for the symptoms of subacute combined degeneration of the cord, at an average interval of twelve months from their discharge. Only two (6 per cent.) are dead; one did not take the diet after discharge, the other took it regularly until he died (aged 66) fifteen months later.

2. Of the 47 patients who were not prescribed the liver diet 50 per cent. are dead, at an average interval of ten months from discharge. The remainder, almost without exception, are now on the diet. Their progress is being watched.

3. The patients who have done best are those who have taken the liver raw or half-raw. Those who have eaten it well cooked have not done so well.

4. A history of tingling and numbness of the fingers and toes, presumably the earliest symptoms of involvement of the cord, was given by 44 per cent. of all our patients. Of those that are still alive and on liver trestment 52 per cent. have no such symptoms now; 11 per cent. are better, and the remainder (almost all of whom had alterations from the normal reflexes) are no better or are definitely worse. This suggests that liver treatment has no effect on the symptoms of subacute combined degeneration of the cord, once the reflexes show that there is organic disease in the central nervous system. The symptoms gradually become worse, although the patient's bodily health is much better.

5. Relapses do occur even when the patients are taking liver regularly, but they are not nearly so common (only six cases noted in our series) as they were before liver treatment was tried. Five of these cases were taking the liver well cooked, the sixth was taking it raw, but the relapse followed an attack of influenza.

6 . Of the 47 cases of pernicious anaemia sent out without liver, only 2 had ever worked regularly before the advent of the liver diet; whilst of the 33 cases sent out on the diet 14 are back on full work and 5 are doing part-time work-a total of 57 per cent. at work.

7. During their periods as in-patients (an average of forty-five days in each case) the following alterations in the constituents of the blood were noted.

\begin{tabular}{ll|c|c|c}
\hline & Erythrocytes. & Leuc cytes. & Haemog'obin. \\
\hline Patients not on liver &.. & $+46 \%$ & $-8 \%$ & $+49 \%$ \\
Patients on liver & $\ldots$ & $+91 \%$ & $+41 \%$ & $+77 \%$ \\
\hline
\end{tabular}

Of the patients who have done well, almost all have retained some degree of anisocytosis and poikilocytosis; all have abundant platelets, and in all the staining of the erythrocytes is normal.

8. Diarrhoea and gastric disturbances appear to have been cured by the liver treatment, only one case of recurrent diarrhoea having been noted in a patient who is otherwise quite well. There are no indications that treatment by hydrochloric acid has any appreciable infivence-most -tients discontinuing its use after discharge. Inadequato liver diet, however, frequently causes a return of gastric discomfort.

9. Reinvestigation of the, condition of the stomach by fractional test meals shows the following results:

(a) Achlorhydria persists in nearly every case. Only two exceptions have been noted, and these will be discussed in a later paper.

(b) The total acidities of all samples withdrawn are greatly diminished, being equivalent to 1.5 to $10.9 \mathrm{c.cm}$. (average 4 c.cm.) of N/10 alkali per $100 \mathrm{c.cm}$. gastric contents.

(c) Total and mineral chlorides, whilst showing parallel titres, give widely varying values in different cases.

(d) Peptic activity is very considerably diminished or negligible.

(e) The fasting contents in over 80 per cent. of the cases are less than $38 \mathrm{c.cm}$., the remainder falling between 50 and $80 \mathrm{c.cm}$.

$(f)$ In practically all cases mucus is present in very marked amount in the fasting contents and frequently in the earlier samples of the test meals.

(g) Lactic acid is invariably absent.

(h) The stomach empties on an average in one to one and a quarter hours.

10. Several sequelae of the diet have been described in the literature. Our observations on them are as follows:

(a) Persisting eosinophilia (5 to 14.75 per cent.) occurs in patients taking the liver diet.

(b) Albuminuria has never been found.

(c) One case of venous (femoral) thrombosis has been observed.

We have to thank the physicians of the Manchester Royal Infirmary for permission to use their cases in the course of this investigation.

\section{BRUCELLA ABORTUS INFECTION IN MAN. Report of a Case.} BY

IVOR J. DAVIES, M.D., F.R.C.P.LoND., ASSISTANT PHYSICIAN, CARDIFF ROYAL INFIRMARY. AND

W. CAMPBELL ANDERSON, M.D., B.Sc., D.P.H., CARDIFF.

IT has now becn definitely proved that infection from the organism known as the Brucella abortus Bang, the cause of contagious abortion in cattle, may occur in human beings. Cases have occurred in America, Rhodesia, Italy, and Germany, with identification of the organism. It is probable that this infection in man is not rare, and if the possibility be borne in mind its incidence may perhaps be found to be not uncommon. In this country many likely cases have been recorded, but in which absolute proof through identification of the organism was absent.

Theodore Thompson ${ }^{1}$ has recently published a report of a case of this infection in this country; he also presented a review of the literature concerning $B$. abortus infection in man and animals, and noted the similarity between B. abortus and $M$. melitensis and their associated diseases. Thompson ${ }^{1}$ stated that this was the seventh authentic instance of the infection to occur in this country. Manson-Bahr, ${ }^{2}$ Bamforth, ${ }^{3}$ and Wordley ${ }^{4}$ havo also reported cases of undulant fever in which the blood agglutinated $B$. abortus or $\boldsymbol{M}$. melitensis in high dilutions, but failed to demonstrate the actual organism. H. Harrison and G. S. 
Wilson, ${ }^{5}$ in an examination of 998 serums sent for Wassermann testing, and negative to this reaction, found when the serum was tested against $B$. abortus that 5.5 per cent. agglutinated this organism; the average titre was 1 in 64 . The proportion of females to males proving positive was nearly two to one. A titre of 1 in 40 was not uncommonly net with, and is probably of no significance in the diagnosis of abortus fever. Forty-two scrums sent in for Widal testing, and proving negative to members of the typhoidparatyphoid group, have been examined for agglutinins to 73 . abortus ; 26.2 per cent. agglutinated this organism to a titre of 1 in 10 or higher. The average titre was 1 in 336. The proportion of females to males proving positive was nearly two to one.

Harrison and Wilson ${ }^{5}$ advanced the tentative conclusion that 33. obortus is probably capable of infecting human beings in this country; and it is suggested that blood cultures and agglutinin tests should be made on all cases of undiagnosed pyrexia and of indefinite, occasionally febrile, maladies. A reference was made in their paper to an important contribution by $\mathbf{M}$. Kristensen ${ }^{6}$ of Copenhagen. He cxamined 1,177 Widal serums and found that 89 of them agglutinated B. abortus to 1 in 100 or higher. Blood cultures were made from 20 of these patients, and in 13 of them an organism indistinguishable from B. abortus was isolated. Of the 89 patients 68 were males and 21 females; no patient was under 13 years of age. Clinically in Scotand. He was very tired at the end of the journey, but soon recovered, and enjoyed his holiday. On July 27 th and 28 th he felt much fatigued after a round of golf, and this was particularly noticeable as it came at the end of a good holiday. He was again tired after the return journey, and was not in his usual good health throughout September. During this month, and for some time before his holiday, he had been subject to eructations and flatulent discomfort. At home he usually consumed daily about a pint of a so-called certified milk, and occasionally small amounts of cream.

The acute stage of the illness commenced on October 2nd, through an abrupt onset resembling that of influenza, with general pains, headache, and fever, but without evidence of catarrh. The temperature was taken daily for a week, but not recorded, though it was raised throughout, and medical advice was not sought until later. Sweats of moderate severity followed the fover fairly regularly in the evenings, with an occasional feeling of chill just before the rise of temperature. He improved, and returned to his work for four days when his temperature was normal, but a feeling of malaise remained. A further fourteen days' holiday was spent at Bournemouth. The temperature was daily subnormal throughout this period, and some general improvement resulted. He returned home on October 7 th, and was again fatigued; the temperature on the evening of this day rose to $100^{\circ} \mathrm{F}$., and the acute stage of his illness lasting six weeks followed. The record of the fever was not commenced until November 4th, but during the previous week the temperature was regularly a little over $100^{\circ} \mathrm{F}$. in the evenings and almost normal in the morning.

The Pyrexia.-The course of the temperature was that of an almost regular intermittent fever (see chart), most of ten $101^{\circ}$

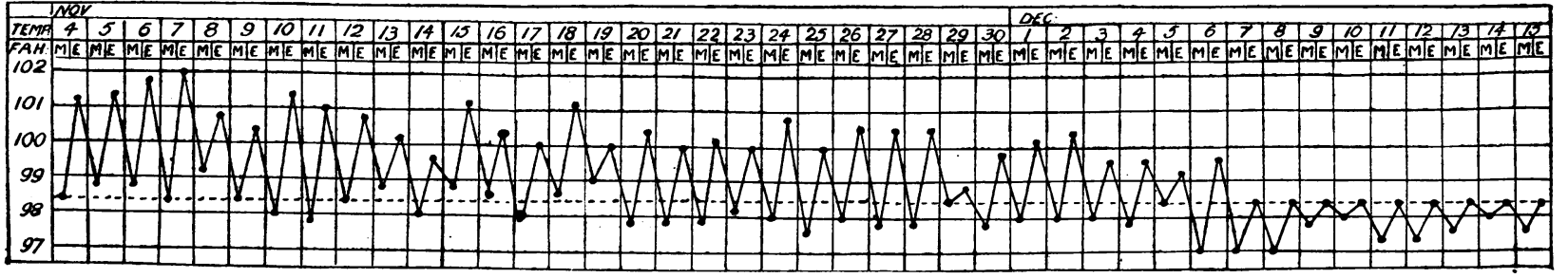

Chart to Show the Course of Five Weeks' Intermittent Fever.

the picture was that of undulant fever, with sometimes severe sweating, localized pains, bronchitis, diarrhoea, or enlarged spleen. Infection occurred apparently from contact with aborting cows or from drinking raw milk or cream.

R. A. Kern ${ }^{7}$ of Philadelphia has made an admirable contribution on the clinical aspects of abortus infection, and concluded that it was widely prevalent in America. He stated that surveys have shown that up to 90 per cent. of herds are infected in some regions, and that practically no region is free. Infectious abortion has long been a serious economic problem in the dairy industry of America. To the question, In view of such extensive bovine infection, why so few human cases?-fewer than 40 are on record in his country-Kern replies that many cases have been overlooked because, owing to the variability of the disease, there is no characteristic clinical picture. He quotes Bassett-Smith ${ }^{8}$ to the effect that Malta fever, because of its variety of forms, is seldom recognized in its early stages in the countries in which it is known to be endemic; most of the patients are treated for something else at first. Kern remarked that if this is true of Malta fever in its normal habitat, how much more likely will the bovine type of the disease be overlooked. He believes that there is as yet a very low index of clinical suspicion of the presence of the disease on the part of general practitioners, and as a result many cases are, in all likelihood, going undiagnosed. These comments are particularly valuable as only 7 cases have been reported in this country. Kern concludes that a clinical consciousness of the disease will lead to the routine testing for Brucella agglutinins in all undiagnosed fevers.

\section{CASE REPORT.}

Male, aged 44 years, director of music, robust, active, and fond of outdoor pursuits. He was of careful habits, except for a partiality to raw milk and cream. He had never lived abroad, and had not been out of this country for several years. On July 13th, 1928, he motored to Scotland from Wales, and spent a fortnight's holiday touring in the Highlands. Raw milk and cream were consumed at several places on the way, at farms in the country, and dairies in the cities, and also daily throughout his stay for the first three weeks, and slightly lower afterwards. The reading was frequently normal or subnormal in the morning, rising as a rule at 5 p.m., and highest at 7.30 p.m., and of ten fell to $100^{\circ}$ or $99^{\circ}$ a few hours later. The highest temperature recorded was $102^{\circ}$ at 5 p.m. on November 7 th, but this reading was not repeated, and was probably influenced by the extraction of a few teeth. The fever was thus continuous over a period of six weeks without any afebrile intervals, except for one day towards the end of the fifth week of the acule stage. An afebrile period of over fourteen days' duration occurred soon after the onset, as has already been described. Profuse sweats, often drenching, which had a peculiar and disagrecable odour, occurred almost daily. Shivering attacks were not infrequent, and, more especially in the early stages, with a few rigors. Headache of some severity was complained of only at the beginning of the illness, being only slight afterwards. Pain was not a feature, and was absent except at the onset; slight joint pains were felt only in an early stage, before he was confined to bed. Constipation had been present for some time previously, and throughout the febrile stage, but was easily controlled.

Physical cxamination was almost negative. The tongue was thickly coated during the whole course of the fever. Much conservative work had been done in the mouth, and the gums appeared to be generally unhealthy. The abdomen was a littlo distended, but free from tenderness, and the liver and spleen were not palpable. No glands were felt. There was no rash. The heart and lungs were normal, except that the heart sounds lacked tone. The pulse rate on an average was 120 per minute during the course of the disease, and was very easily accelerated afterwards in the convalescence, and noticed by the patient. The blood pressure on November 6th was $130 \mathrm{~mm}$. systolic and $80 \mathrm{~mm}$. diastolic. Respiration was elevated with the rise of temperature but was otherwise normal, and no bronchial catarrh was present at any time. The urine was normal, except for a transient siight glycosuria about the middle of the acute period. A diagnosis of oral scpsis was first made, and radical dental treatment was carricd out on November 7th and in the week which followed. A streptococcal infection was considered likely as a susceptibility was assumed, owing to the occurrence in 1925 of an ulcer on the lateral surface of the tongue, which remained indolent for three months before healing took place. The Wassermann reaction at that time was negative.

The course of the temperature was unaffected by the dental treatment. The gums remained inflamed, spongy, with slight suppuration, until on December 1st a small, foul-smelling bit 
of necrosed bone extruded, and healing of the mouth afterwards gradually followed. The temperature dropped to normal within a week of the separation of the necrosed bone, and no further attacks of fever have arisen up to the time of the report. The patient lost $14 \mathrm{lb}$. in weight in the course of the illness. Convalescence was slow, and five months later he had not fully regained his usual health, and had only recovered $2 \mathrm{lb}$. in weight. The tongue remained slightly furred, and he complained of precordial discomfort and aching in the region of the left shoulder. The heart's action was still impaired.

Clinical Pathology.-Examination of the blood on November 23rd showed no qualitative change in the red cells or malarial parasites. The blood count was as follows:

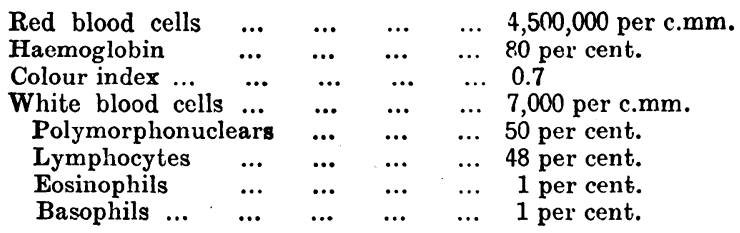

Agglutination Tists.-On November 23rd the patient's serum was negative to the typhoid-paratyphoid group, but positive to the Brucella abortus in $\varepsilon$ dilution of 1 in 250 . A week later the positive agglutination reaction to this organism had risen to 1 in 800, and this titre was maintained on each of the two following weeks. On March 20th, 1929, after three months' convalescence, the agglutination reaction to the Brucclla abortus was completely positive in a dilution of 1 in 1,000. The tests were repeated on May 28th, when the agglutination reaction was found to be still positive, but the titre had now dropped to 1 in 200. The blood count was almost unchanged, except that the leucocytes had risen to 9,600 per c.mm., polymorphonuclears 50 per cent., lymphocytes 44 per cent. The leucopenia had thus disappeared, but the relative lymphocytosis remained.

Blood culture and urine culture were negative on December 13th, 1928. The Wassermann reaction was negative.

\section{Diagnosis.}

The diagnostic problem, as $\mathrm{Kern}^{7}$ remarks, is eminently one for the laboratory. The only solution lies in the routine use of the appropriate tests in all undiagnosed fevers. Hardy ${ }^{9}$ has suggested that, until physicians generally are cognizant of the disease, all serums sent to State and municipal laboratories for Widal's test should be tested for the Brucella as well.

Blood culture and serum agglutination tests are essential. Urine culture may also be of value. The blood count in a case of Brucella abortus infection is that of a leucopenia with a relative lymphocytosis. There is no characteristic clinical picture. Fever is the only universal symptom, but there is no distinctive curve which may be continuous or undulatory with intervening afebrile periods. The continuous fevers may be sustained or remittent to an extent simulating that of septic infection or malaria. Some patients become progressively ill and soon seek advice; others are little disturbed by the infection, and may not take to bed at once, and then only for a brief period of the duration of the disease, or they may be wholly ambulatory. Kern ${ }^{7}$ submits an important clinical observation: "One gets the impression rather generally that the patients are subjectively not so ill as their appearance and fever may seem to warrant." The patient whose case is now reported did not believe himself to be ill enough to warrant the services of a nurse.

\section{Differential Diagnosis.}

Bracella infection in symptomatology closely simulates that of other diseases, but, if borne in mind, suitable tests will usually be decisive. Septicaemia from hidden foci of infection must in particular be considered; also the typhoid-paratyphoid group, $B$. coli infection, tuberculosis, subacute bacterial endocarditis, leukaemias, pernicious anaemia, Hodgkin's disease, and malignant disease. A careful review of the previous health and habits, mode of onset, course of the disease, routine physical examination, and a close analysis of laboratory tests will rarely fail to lead to a definite diagnosis.

The case of Scott and Saphir ${ }^{10}$ is of great interest because of the association with an infective endocarditis engrafted on an old mitral lesion. Blood culture yielded the Brucella abortus, and the serum agglutination reaction was positive in 1 to 1,000 dilution. At no time were streptococci found in the blood. The heart's blood yielded the abortus organism but no streptococci. The morbid anatomy was typical of infective endocarditis. No other source of infection was found. There was no actual proof that the abortus organism was responsible for the acute endocarditis, and the case was reported as "one of acute and chronic endocarditis associated with Brucella melitensis (abortus) bacteriaemia." It remains to be shown whether this organism will ultimately find a place in the bacteriology of infective endocarditis.

\section{Treatment.}

This is mostly symptomatic. Mercurochrome has been tried and appears to have been curative in a few cases. The drug is given intravenously in doses of 10 to $20 \mathrm{c.cm}$. of a 1 per cent. solution, and can be repeated once or twice if necessary after an interval of sereral days.

\section{Prophylaxis.}

Infection of human beings by the Brucella abortus organism mainly arises through the drinking of raw milk. The remedy appears to be obvious, but probably incapable of universal adoption. The supply of milk is only second in importance to the water supply, and should be solely under the control of the municipal authorities, and not left to the caprices of private enterprise. Various methods of herd prophylaxis and treatment of infected animals have been proposed. Birch ${ }^{11}$ recommends the detection and segregation of all infected cattle. Karsten ${ }^{12}$ in Germany (quoted by $\mathrm{Kern}^{7}$ ) makes several valuable recommendations, and amongst others: (1) Blood agglutination tests in all animals in the herd, including the bull once a year. Reacting animals are to be killed or segregated. (2) In freshly infected herds in which less than 10 per cent. of the animals are involved, the disease is eradicable by killing the infected individuals, repeated disinfection of stables, and routine testing of the remaining cattle. $\mathrm{He}$ feels that these more radical measures are preferable to the therapeutic vaccination when the incidence of infection in a herd is low.

In Rhodesia infectious abortion in cattle has been made a notifiable disease. Infected herds are quarantined until six months after the last abortion or until satisfactory vaccination has been carried out; stables are disinfected and reacting bulls are killed. Vaccine treatment of segregated infected animals seems to meet with fair success.

\section{Summary.}

The case here reported is almost certain to be one of infection by the Brucella abortus; it is the eighth to be reported in this country, and the first from Wales. Actual isolation of the organism (for example, from the blood) would of course be necessary for absolute proof, but the clinical details are closely like those of published reports in cases where the organism was actually identified. The duration was at.least nine weeks; it is impossible to be exact owing to the uncertain time of onset, as was also shown in a majority of the published cases.

The case was one of prolonged pyrexia of unknown origin until the blood was submitted to the agglutination test against the Brucella abortus organism, when the cause of the infection was discovered. The serological test was positive in high dilution. No local source for the infection could be traced.

We are much indebted to Dr. W. Parry Morgan, bacteriologist to the Cardiff and County Public Health Laboratory, for tho pathological investigation.

REFERENCES.

1 Thompson, T. : Lancet, 1928, ii, 1335. ${ }^{2}$ Manson-Bahr, P.; Ibid., 1927, ii, 1414. 3 Bamforth, J.: Ibid., 1927, i, 818. * Wordley, E. : Ibid., 1927, ii, 1290. 5 Harrison, H., and Wilson, G. S. : Ibid., 1928, ii, 1338. 6 Kristensen, M. : Centralbl. f. Balt., 1928, cviii, 89. 7 Kern, R. A. : Amer. Journ. Med. Sci., 1928, clxxvi, 405. ${ }^{8}$ Bassett-Smith, P. W.: Journ. Roy. Tacal Met. Serv., 1927, 13, 258. 'Hardy, A. V.: Publio Health Reports, 1928, 43, 503. 10 Scott, R. W., and Saphir, 0. . Amer Journ. Med. Sci., 1928, 175, 66. ${ }^{11}$ Birch, R. R. : Cornell Vet., 1927, 17, 221. 12 Karsten : Deut. Tierürzt Woch., 1927, 35, 201. 